\title{
Revisión taxonómica de los géneros Oratha Walker 1863 y Hasodima Butler 1882 (Lepidoptera: Geometridae)
}

\author{
Taxonomic revision of the genera Oratha Walker 1863 and Hasodima Butler 1882 \\ (Lepidoptera: Geometridae)
}

\section{LUIS E. PARRA ${ }^{1} \&$ MARTA PASCUAL-TOCA ${ }^{2}$}

\begin{abstract}
${ }^{1}$ Departamento de Zoología, Facultad de Ciencias Naturales y Oceanográficas, Universidad de Concepción, Casilla 160-C, Concepción, Chile; e-mail: luparra@udec.cl

${ }^{2}$ Becaria Intercampus, Departamento de Biología de Organismos y Sistemas, Unidad de Zoología, Facultad de Biología, Universidad de Oviedo, C/Catedrático Rodrigo Uría s/n, 33071 Oviedo, España; e-mail:mpascual@correo.uniovi.es
\end{abstract}

\begin{abstract}
RESUMEN
Los géneros Oratha Walker 1863 y Hasodima Butler 1882 del sur y centro de Chile son revisados y sus especies redefinidas y redescritas. Las especies quedan como sigue: Oratha significata Walker 1863, Hasodima elegans Butler 1882, H. boreas (Butler 1882) comb. nov. y H. bartletti nov. sp. El análisis de los tipos y de la genitalia permite colocar Aconcagua fessa Rindge 1975 en la sinonimia de Aconcagua parva (Butler) comb. nov. Se describe e ilustran los adultos, las armaduras genitales y la distribución de las especies. Se entrega una clave para separar las especies de Hasodima. La monofilia de los géneros y sus relaciones con taxa cercanos se discute brevemente.
\end{abstract}

Palabras clave: Ennominae, Ourapterygini, Aconcagua fessa, Boletobia sericea, taxonomía, Chile.

\begin{abstract}
The genera Oratha Walker 1863 and Hasodima Butler 1882 from central and southern Chile are redefined and its species revised and described. The species are Oratha significata Walker 1863, H. elegans Butler 1882, H. boreas (Butler 1882) and $\mathrm{H}$. bartletti sp. nov. The analysis of the types and the genitalia allow placing Aconcagua fessa Rindge in the synonymy of Aconcagua parva (Butler) comb. nov. All species and their genitalia and distribution are described and illustrated. A key for species of Hasodima is provided. The monophyly of the genera and its relationships to the allied taxa are briefly discussed.
\end{abstract}

Key words: Ennominae, Ourapterygini, Aconcagua fessa, Boletobia sericea, taxonomy, Chile.

\section{INTRODUCCIÓN}

Este trabajo es parte de una serie de revisiones que se están realizando en representantes de la familia Geometridae que se distribuyen en los bosques templados del sur de Chile y Argentina. El fin es aclarar la situación sistemática y contribuir con el conocimiento de la biodiversidad de esta región del mundo. Esta zona es de especial interés por corresponder a un área con un prolongado historial de aislamiento geográfico, traducido en la presencia de taxa con altos niveles de endemismo, relictos y/o que han retenido caracteres ancestrales (Armesto et al. 1996, Villagrán \& Hinojosa 1997). La intención es clarificar las relaciones de parentesco de los miembros de esta familia, sus respectivas tribus y la importancia de este aislamiento en la evolución de los diferentes taxa de Geometridae.
Ennominae es la subfamilia más diversa de Geometridae y está dividida en numerosas tribus, pero su clasificación superior aún permanece no resuelta (Scoble 1995). Las tribus más diversas y con una distribución más amplia corresponden a Lithinini y Nacophorini, constituyéndose en el foco de atención en varios estudios en los últimos años (Rindge 1983, Rindge 1986, Parra 1997, 1999a, 1999b). Sin embargo, otras tribus con una distribución conocida para la región Neotropical, representadas en menor proporción en la región Neártica, presentan varios géneros cuya taxonomía aún permanece no resuelta. Las tribus Anagogini, Cingiliini y Ourapterygini (Forbes 1948) tienen muchos caracteres en común en los diferentes estados de desarrollo (McGuffin 1987), condición que las hace difícil de diferenciar, por ello el análisis y estudio de los taxa que comparten estas características son de especial importan- 
cia para obtener caracteres diagnósticos que permitan distinguir ambas tribus o establecer las sinonimias correspondientes.

En nuestro estudio de la tribu Ourapterygini en Chile, hemos encontrado que existe confusión taxonómica entre las especies que forman parte del género Oratha Walker 1863. Oratha fue propuesto inicialmente para incluir sólo a Oratha significata Walker (1863). Bacillogaster boreas y B. parva, ambas descritas por Butler (1882), fueron transferidas junto con Boletobia sericea (Butler 1882) al género Oratha, por Scoble (1999). Butler (1882) describe el género Hasodima para la especie $H$. elegans de Chile, la cual muestra mucha semejanza externa con las especies de Oratha. Ambos géneros no han sido revisados, por tanto el objetivo de este trabajo es aclarar la situación taxonómica de ambos y obtener los caracteres diagnósticos entre ambos y otros taxa relacionados. Esta revisión incluye los cambios taxonómicos oportunos tras el estudio de los caracteres morfológicos en ambos sexos.

\section{MATERIALES Y MÉTODOS}

Para el presente estudio se contó con el material depositado en el Museo Nacional de Historia Natural, Santiago, Chile (MNHN), Museo de Zoología
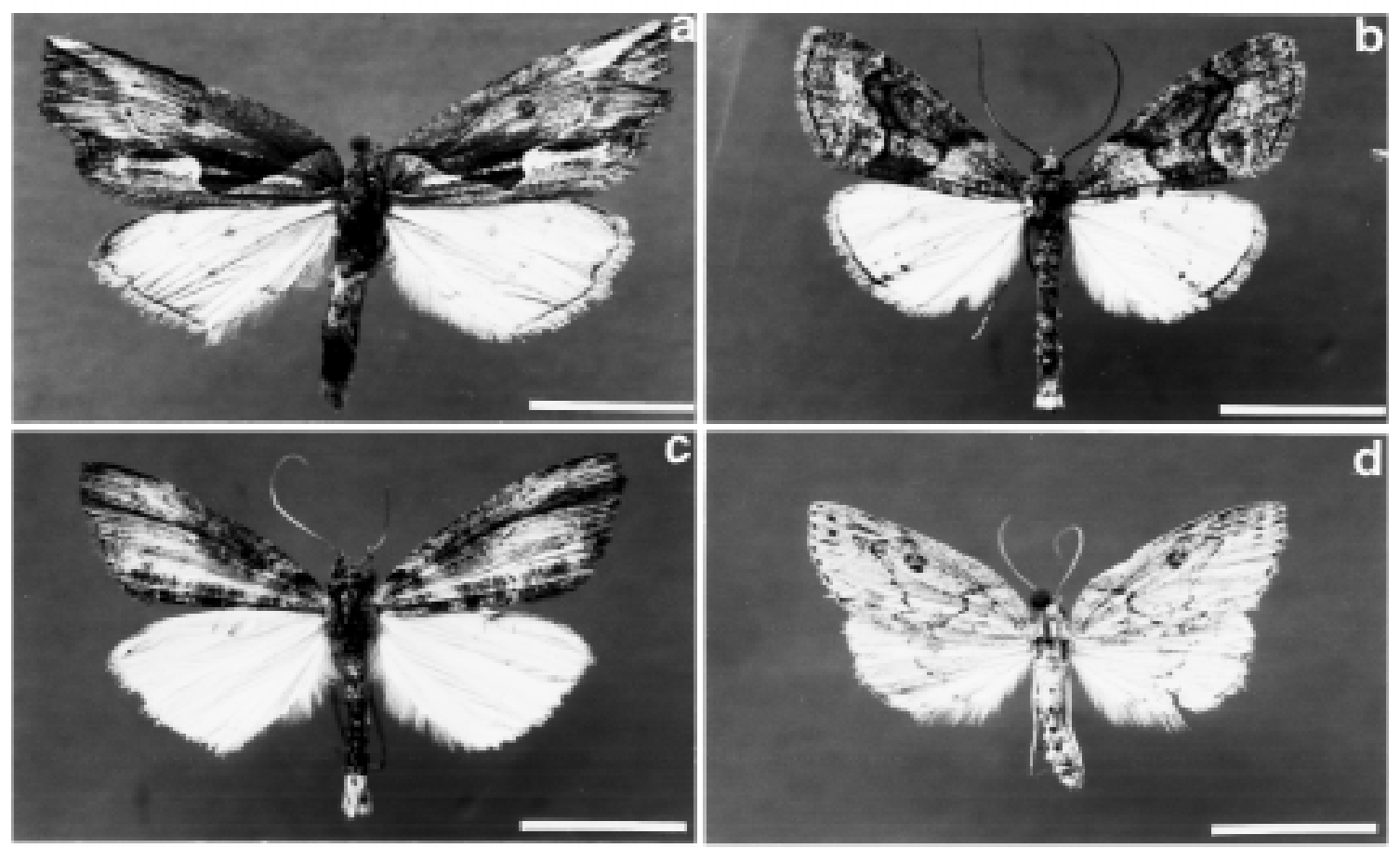

Fig. 1: Imagos de las especies de Oratha y Hasodima: (A) O. significata; (B) H. elegans; (C) $H$. boreas; y (D) H. bartletti. Escala: $1 \mathrm{~cm}$.

Adults of the Oratha and Hasodima species: (A) O. significata; (B) H. elegans; (C) H. boreas; and (D) H. bartletti. Scale: $1 \mathrm{~cm}$. 
del gnathos y el patrón de las manchas en las alas anteriores (Fig. 1A), también constituyen caracteres diagnósticos. En Oratha las manchas sobre las alas no forman las típicas bandas transversales como en los otros géneros de la tribu. Hemiloxia Warren y Oratha tienen la región dorsal del uncus engrosada, pero a diferencia de Hemiloxia, el engrosamiento del uncus en Oratha se debe a la presencia de una cresta bilobulada con cerdas.

Monofilia. La monofilia del género está sustentada por las siguientes sinapomorfias: (1) uncus con cresta dorsal bilobulada y cubierta por una fila de cerdas; (2) gnathos en forma de "U" con espinas gruesas; y (3) furca cuneiforme.

Redescripción: imago. Antenas en los machos y hembras simples. Alas anteriores de color castaño oscuras con las bandas antemedial, postmedial y terminal presentes, delimitando en ocasiones zonas más oscuras. Mancha discal siempre presente, más o menos aparente. Alas posteriores ocres, con mancha discal y banda terminal presentes. Tibias posteriores del macho y la hembra con dos pares de espolones; pincel de pelos en la tibia posterior del macho. Abdomen de color similar a las alas, con penachos de escamas en varios de sus segmentos. Genitalia del macho (Fig. 2A y 2B). Uncus en forma de gancho, con una cresta bilobulada en su región dorsal; socii pequeño, globular y con cerdas; gnathos en forma de "U"; anellus cóncavo basalmente; proceso del anellus medial, aunque pueda tener además procesos laterales. Valvas con o sin estructura secundaria, con costa esclerosada y una escotadura apical en la zona interna. Saccus redondeado. Aedeagus con vesica armada con espinas. Genitalia de la hembra (Fig. 2c). Corpus bursae globoso, con la parte anterior membranosa y la posterior esclerosada y con surcos; signum pedunculado y lanceolado; apófisis posteriores de 3 a 4 veces mayor que las anteriores; ductus bursae cuadrangular o rectangular, con las áreas laterales esclerosadas de 5 a 10 veces menor que el corpus bursae.
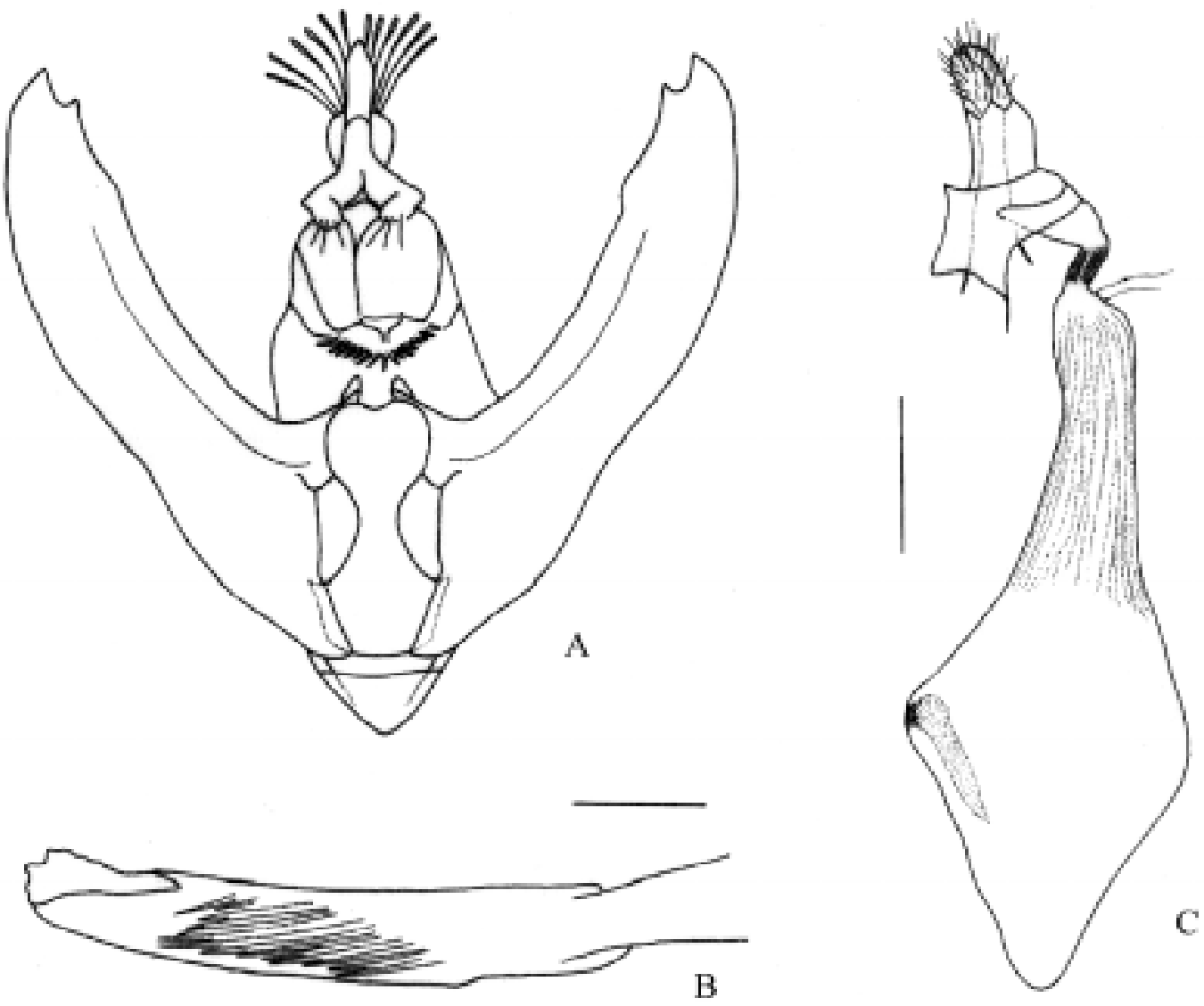

Fig. 2: Armaduras genitales del macho y la hembra de $O$. significata: (A) genitalia del macho en vista ventral; (B) aedeagus en vista lateral; (C) genitalia de la hembra en vista lateral. Escala: $1 \mathrm{~mm}$.

Male and female genitalia of $O$. significata: (A) male genitalia in ventral view; (B) aedeagus in lateral view; (C) female genitalia in lateral view. Scale: $1 \mathrm{~mm}$. 
Distribución. Su única especie se distribuye en la zona Central y Sur de Chile (Fig. 6).

Observaciones. El género fue incluido originalmente en la familia Pyralidae y con posterioridad (Fletcher 1979) es ubicado correctamente en la familia Geometridae.

Oratha significata Walker 1863 (Fig. 1A, 2A, 2B, 2C)

Oratha significata Walker 1863, p. 15; Scoble (1999), p. 674.

= Alsophilla ? hypparia Felder \& Rogenhofer

1875, Plate 2, Fig. 35; Butler 1882, p. 393

(Bacillogaster); Butler 1894, p. 520.

Tipos. Oratha significata, Holotipo $\bigcirc$, Chile, BMNH (examinado); Alsophilla? hypparia, Sintipo $\bigcirc$, Chile, BMNH (examinado).

Redescripción: macho (Fig. 1A). Antenas simples, ciliadas, superficie dorsal castaño oscura hacia el extremo proximal y castaño claras hacia el extremo distal. Frente y palpos maxilares castaño claros. Patagias castaños oscuras, ápice de las escamas blancas. Tégulas con escamas castaño claras en su superficie y borde castaño oscuro. Tórax y patas castaño claros; fórmula tibial 0-24. Alas anteriores castaño claras; con una "Y" castaño oscura en la base del ala, cuyas ramas son paralelas a las venas Sc y A, respectivamente; banda antemedial castaño oscura, inclinada hacia el borde exterior para unirse a la banda postmedial en la región media del margen interno formando un ángulo castaño oscuro; región entre la base y la banda antemedial del ala cenicienta; entre la base de la banda postmedial y el margen externo de ala hay una mancha subcircular cenicienta que se continua con una franja castaño oscuro hacia el margen externo; mitad anterior del ala castaño ceniciento; mancha discal castaño claro; ápice del ala con una mancha triangular cenicienta; banda terminal castaño oscuro; región ventral con la mancha discal y la banda terminal castaño oscuro. Ala posterior castaño ceniciento; mancha discal y banda subterminal tenuemente marcadas, con la banda terminal oscura; región ventral del mismo color, con la mancha discal más notoria. Abdomen castaño ceniciento; con penachos de escamas castaños oscuros en la región dorsal de los segmentos abdominales 1 al 5; penacho del segmento 2 muy conspicuo. Genitalia del macho (Fig. 2A y 2B). Uncus recto, lanceolado, con una protuberancia dorsal bilobulada, la que lleva una hilera de cerdas en la zona apical. Socii reducido, con cerdas en el extremo. Gnathos en forma de "U", extensión central subcuadrangular armada de numerosas espinas. Anellus en su zona basal subcuadrangular; furca cuneiforme con el margen anterior emarginado. Valva sencilla, con una hendidura apical en forma de media luna, de longitud subigual a tres veces la longitud del anellus. Saccus subtriangular. Aedeagus recto, 1,5 veces más largo que el anellus; vesica con microespinas, midiendo las más largas un tercio de la longitud total del aedeagus.

Hembra: similar al macho; tibias posteriores sin pincel de pelos. Genitalia de la hembra (Fig. 2C). Corpus bursae con zona anterior hinchada y membranosa, terminado en una proyección redondeada; zona posterior esclerosada con estrías longitudinales. Signum grande, pedunculado, lanceolado con microespinas, cinco veces menor que el corpus bursae. Apófisis posteriores casi tres veces más largas que las anteriores. Ductus seminalis nace en el límite entre el corpus bursae y el ductus bursae. Ductus bursae con las áreas laterales esclerosadas, de forma cuadrangular, 10 veces menor que el corpus bursae.

Longitud del ala anterior. Desde la base al ápice: de 17 a $18 \mathrm{~mm}$ para los machos y de 17 a 20 $\mathrm{mm}$ para las hembras.

Distribución. Esta especie se distribuye entre $\operatorname{los} 33^{\circ}$ y $\operatorname{los} 39^{\circ} \mathrm{S}$, entre las provincias de Santiago y Cautín (Fig. 6).

Periodo de vuelo. Con presencia de adultos desde octubre a abril.

Observaciones. La redescripción de la especie se basó en 15 machos y 17 hembras. No se observó variación intraespecífica a pesar de su amplia rango de distribución. Las manchas y colores mantienen un patrón constante en todos los ejemplares estudiados.

Material examinado: (32 ejemplares). Oratha significata, Holotipo 9 , Chile (BMNH); Alsophilla? hypparia, Sintipo $\bigcirc$, Chile, (BMNH). Valparaíso. Viña del Mar: 1Q, 12-11-1953; 1@, 30-10-1953; 2O0, 15-10-1953 (MNHN). Laguna Verde: 1Q, 13-10-1936 (MNHN). Marga Marga: 19, 9-4-1955 (MNHN). Santiago. Tobalaba: $1 \sigma^{\prime}$, 2-1-1947 (MZUC). Cordillera. Guayacán: 1 , 31-10-1943 (MNHN). 2 ' ర', 24-10-1951, T. Ramir coll. (MZUC). Nuble. Atacalco: 10, 2811-1951, L.E. Peña coll.; 1 $\sigma^{\prime}$, 9-12-1951, L.E. Peña coll. (MZUC). Concepción. Chiguayante: 10, sin fecha, Hulot coll.; 1 $\sigma^{7}$, 28-11-1961, Hulot coll. Concepción: $1{ }^{\top}$, sin fecha; $10^{\top}, 11-11-1954$; $1 \bigcirc, \quad 25-10-1955 ; 1 \sigma^{\top}, 3-11-1958 ; 1 \sigma^{\top}, 24-11-$ 1958; 1ᄋ, 5-11-1960; 1ᄋ, 21-11-1960; $20^{7} \sigma^{2}, 7-$ 12-1960; 1 0 , 6-11-1987. Carretera de Bulnes: km 25: 1 , 27-11-1981. Bio-Bío. La Meseta (río Laja): $1 \gamma^{7}$ y 1@, 27-11-1997 (MZUC). Malleco. Curacautín: 10, 1948; 1@, 15-12-1950 (MZUC). Cautín: Villarrica (P.N. Villarrica): 1Q, 7-11990, L.E. Parra coll. (MZUC). 


\section{Hasodima Butler 1882}

Especie tipo. Hasodima elegans Butler 1882, p. 403. Por monotipia.

Diagnosis. Hasodima se distingue de otros géneros de Ourapterygini por la presencia de un pseudouncus en la genitalia del macho (Figs. 3a, 4 a y 5a) y de un signum pedunculado en la genitalia de la hembra (Figs. 3c y 5c). Hemiloxia maditata (Felder \& Rogenhofer) es muy parecida a las especies de Hasodima, está semejanza se debe a las antenas pectinadas en el macho y al patrón de las máculas en las alas, ambos géneros se pueden distinguir fácilmente por los caracteres de la genitalia del macho y de la hembra.

Monofilia. Las tres especies del género poseen un patrón de coloración y una morfología de la genitalia que sugiere que el género es monofilético. La monofilia del género se sustenta por el carácter sinapomórfico: presencia de pseudouncus en la genitalia del macho.

Redescripción: macho y hembra. Antenas del macho simples o pectinadas, en hembras simples, castaño oscuras o claras en la su región dorsal; frente y palpos maxilares castaño claros o castaño claros abigarrados con escamas castaño oscuras. Tórax con (boreas) o sin penachos de escamas; patagias y tégulas similar en color a las alas anteriores, aveces con sus bordes con una franja castaño oscura (elegans); patas castaño claras; formula tibial 0-2-4; tibias posteriores del macho con un pincel de pelos. Alas anteriores castaño oscuras, claras o castaño verdosas, bandas antemedial y postmedial representadas por líneas castaño oscuro o por bandas claras que cruzan el ala; mancha discal siempre presente (Fig. 1B y 1D). Alas posteriores castaño claras a cenicientas. Abdomen similar en color a las alas anteriores; con penachos de escamas en el dorso de cada segmento abdominal (boreas); en los segmentos 3 al 7 (elegans) y en el tercero (bartletti). Genitalia del macho. Uncus, en forma de gancho, esclerosado, pseudouncus débilmente esclerosado, romboidal (elegans) (Fig. 3A), lanceolado (bartletti) (Fig. 5A) y espiniforme (boreas) (Fig. 4A); socius reducido; gnathos en forma de V, con pocas espinas; valvas estrechas, con la región del cucullus hundida; yuxta reducida, furca
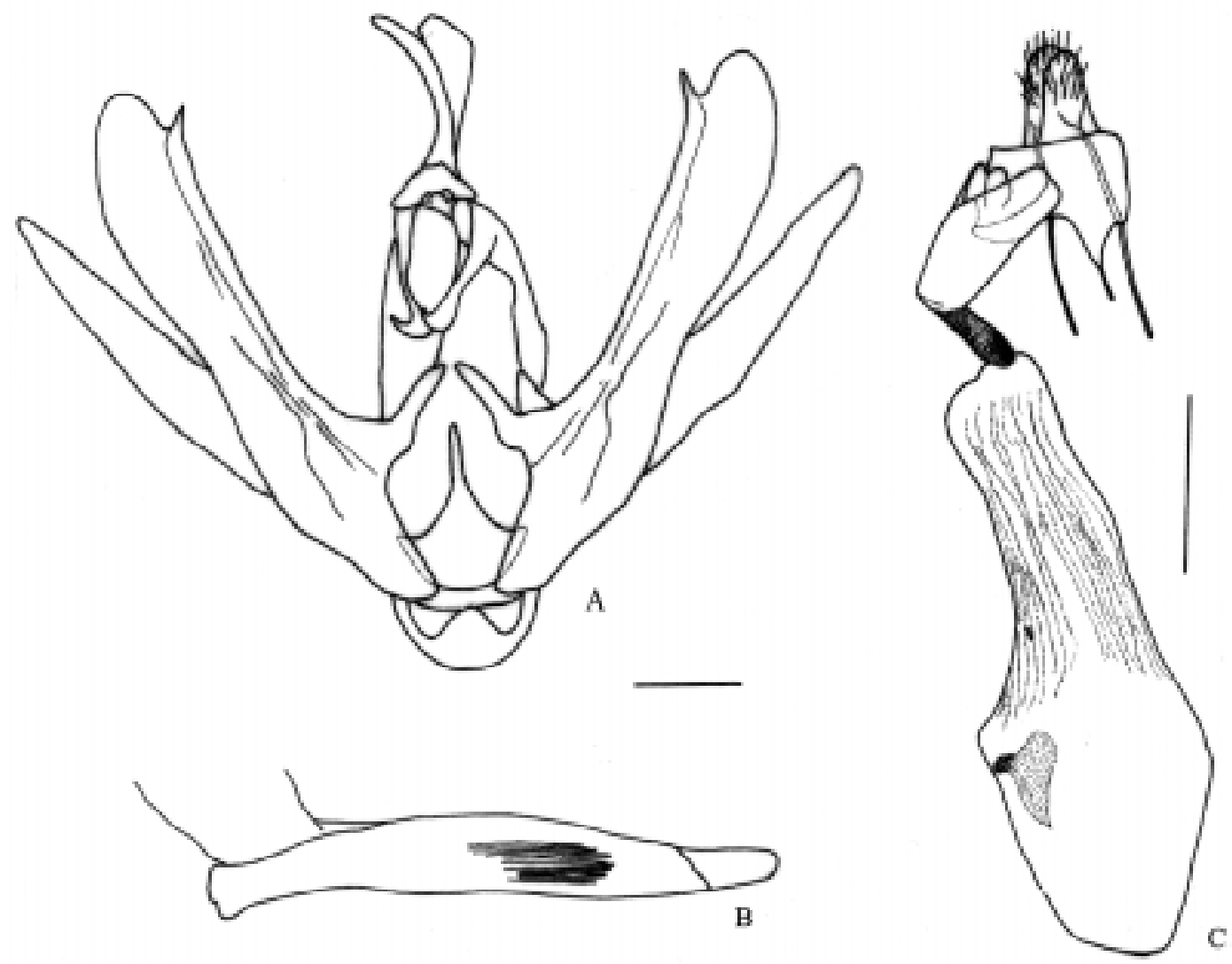

Fig. 3: Armaduras genitales del macho y la hembra de H. elegans: (A) genitalia del macho en vista ventral; (B) aedeagus en vista lateral; (C) genitalia de la hembra en vista lateral. Escala: $1 \mathrm{~mm}$.

Male and female genitalia of $H$. elegans: (A) male genitalia in ventral view; (B) aedeagus in lateral view; (C) female genitalia in lateral view. Scale: $1 \mathrm{~mm}$. 
espiniforme a tubular (boreas); saccus triangulariforme. Aedeagus, vesica con un grupo de espinas o con una área piramidal esclerosada (boreas). Genitalia de la hembra. Región posterior del corpus bursae globoso, membranoso, región anterior tubular, esclerosada y con estriaciones longitudinales; signum presente, pedunculado y sagitado, cubierto de espinas (Fig. 3C y 5C); ductus bursae cuadrangular, fuertemente esclerosado en los bordes; apófisis posteriores tres veces más largas que las anteriores.

Distribución. Los representantes de este género se distribuyen en la zona central y sur de Chile e Isla Mocha (Fig. 6).

Observaciones. Butler describió el género para una sola especie sobre la base de un ejemplar macho. En la descripción original señala que el género pertenece al grupo de géneros de Lobophora Curtis (Larentiinae, Trichopterygini). Los caracteres analizados permiten incluir a este taxón en los Ennominae, como lo muestra Scoble (1999).

Lista sistemática de las especies del género Hasodima: (i) bartletti sp. nov.; (ii) boreas (Butler 1882) comb. nov.; (iii) elegans (Butler 1882).

En el Anexo 1 entregamos una clave de identificación del género Hasodima.

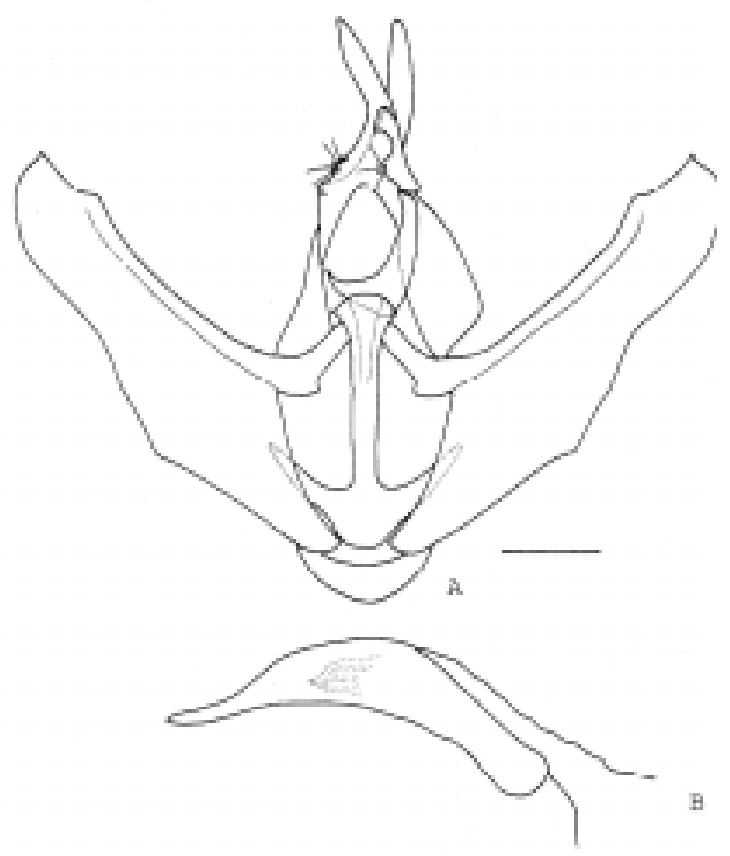

Fig. 4: Armadura genital del macho de H. boreas: (A) genitalia del macho en vista ventral; (B) aedeagus en vista lateral. Escala: $1 \mathrm{~mm}$.

Male genitalia of $H$. boreas: (a) male genitalia in ventral view; (B) aedeagus in lateral view. Scale: $1 \mathrm{~mm}$.
Hasodima elegans Butler 1882 (Fig. 1B, 3A, 3B y $3 C)$

Hasodima elegans Butler 1882, p.403; Butler 1894, p. 532; Angulo \& Casanueva 1981, p. 28; Scoble 1999, p. 422.

Tipo: Hasodima elegans, Sintipo ${ }^{\top}$, Las Zorras, Chile, BMNH (examinado).

Diagnosis. Hasodima elegans se distingue de las otras especies del género por el color castaño verdoso de sus alas anteriores. La genitalia del macho de $H$. elegans se reconoce por la furca espiniforme, pseudouncus romboídeo y la presencia de coremata en las valvas. A diferencia de $H$. bartletti, la armadura genital de la hembra de $H$. elegans tiene la porción anterior del corpus bursae subcónica.

Redescripción: macho (Fig. 1B). Cabeza: antenas bipectinadas, castaño verdosas en su superficie dorsal; frente y vértex castaño verdoso; frente abigarrada con escamas castaño oscuras bajo la antena y el borde del ojo. Tórax castaño verdoso; patagias con el borde distal castaño oscuro; tégulas castaño verdosas; patas con bandas castaño verdosas y claras; formula tibial 0-2-4. Alas anteriores castaño verdosas, bandas antemedial y postmedial fuertemente marcadas en color café, con la zona intermedia algo más oscura que el resto del ala; mancha discal y banda terminal marcadas; superficie ventral castaño claro, marca discal más aparente; inicio de la banda postmedial en la zona costal y banda terminal castaño oscuro. Alas posteriores de color ocre blanquecino; banda postmedial fuertemente marcada en castaño oscuro, se aproxima ligeramente hacia la banda terminal en la base; mancha discal y banda terminal aparentes; superficie ventral ocre con manchas castañas dispersas; banda terminal y banda postmedial marcadas, mancha discal más aparente. Abdomen castaño verdoso en su superficie dorsal, castaño claro a ceniciento en su superficie ventral; penachos de escamas presentes en los segmentos abdominales 3 al 7, similares en color a la superficie dorsal del abdomen y subiguales en tamaño, todos ocupan los $2 / 3$ de cada segmento abdominal. Genitalia del macho (Fig. 3A y 3B). Uncus en forma de gancho; pseudouncus romboídeo; socii reducido, con cerdas; gnathos en forma de "V", con un diente final grande y tres anteriores más pequeños; valvas con coremata en su región dorsal; yuxta redondeada basalmente, furca espiniforme, central y estrecha; valvas con una incisión en la zona apical que forma un pico en el lado interno; presentan estructura secundaria; saccus redondeado; aedeagus recto, vesica con microespinas. 
Hembra. Similar al macho; envergadura alar mayor; con antenas simples; tibias posteriores sin pincel de pelos. Genitalia de la hembra (Fig. 3C). Zona anterior del corpus bursae subcónico, membranoso, con la zona posterior esclerosada y con surcos y la zona anterior membranosa; signum grande, lanceolado con microespinas, cinco veces menor que el corpus bursae; apófisis anteriores aproximadamente dos veces más pequeñas que la apófisis posteriores; ductus seminalis parte de la inserción entre el corpus bursae y el ductus bursae; ductus bursae rectangular, con áreas laterales esclerosadas, cinco veces menor que el corpus bursae.

Longitud del ala anterior, medida de la base al ápice. De 15 a $16 \mathrm{~mm}$ en los machos y de 15 a 18 $\mathrm{mm}$ en las hembras.

Distribución. Esta especie se distribuye entre $\operatorname{los} 33^{\circ}$ y $\operatorname{los} 41^{\circ} \mathrm{S}$, entre la Provincia de Santiago y Llanquihue (Fig. 6).

Periodo de vuelo. Especie recolectada desde el 23 de julio al 15 de enero.
Observaciones. La redescripción de la especie se basó en 10 machos y cinco hembras. Variación intraespecífica se observó en el patrón de coloración de las alas anteriores, especialmente en las áreas basal y terminal del ala. En estas regiones un par de ejemplares provenientes de Osorno y Valdivia muestran un color blanco ceniciento en las alas anteriores.

Material examinado (15 ejemplares). Valparaíso. Las Zorras: 1 Sintipo (BMNH). Santiago. Tobalaba: 10, 10-11-1946; 1 ', 18-111946 (MZUC). Santiago: 1Q, 9-10-1933, Ureta coll. (MNHN); 1 ' 21-10-1959, R. Torre coll. (MZUC). Valparaíso. Viña del Mar: 1 $\sigma^{\prime}, 23-7-$ 1953; 1 , 12-9-1953 (MNHN). La Cruz: 1 Q, 229-1966, J. Aranda coll.; 1 $0^{7}$, 15-1-1974, R. Ripa coll. (MZUC). Linares. Linares: 1O, 15-9-1949 (MZUC). Ñuble. Chillán: 1@, 14-9-1959 (MZUC). Concepción. Concepción: 1 $\sigma^{\top}, 21-10-1960$. Valdivia: Isla Teja: 1 18 , 10-1986, J. Jackson coll. (MZUC). Osorno. Osorno: 10, 21-11-1982, A. Aguilar coll. (MZUC). Llanquihue. Maullín: 1\%, 10-11-1943 (MZUC).
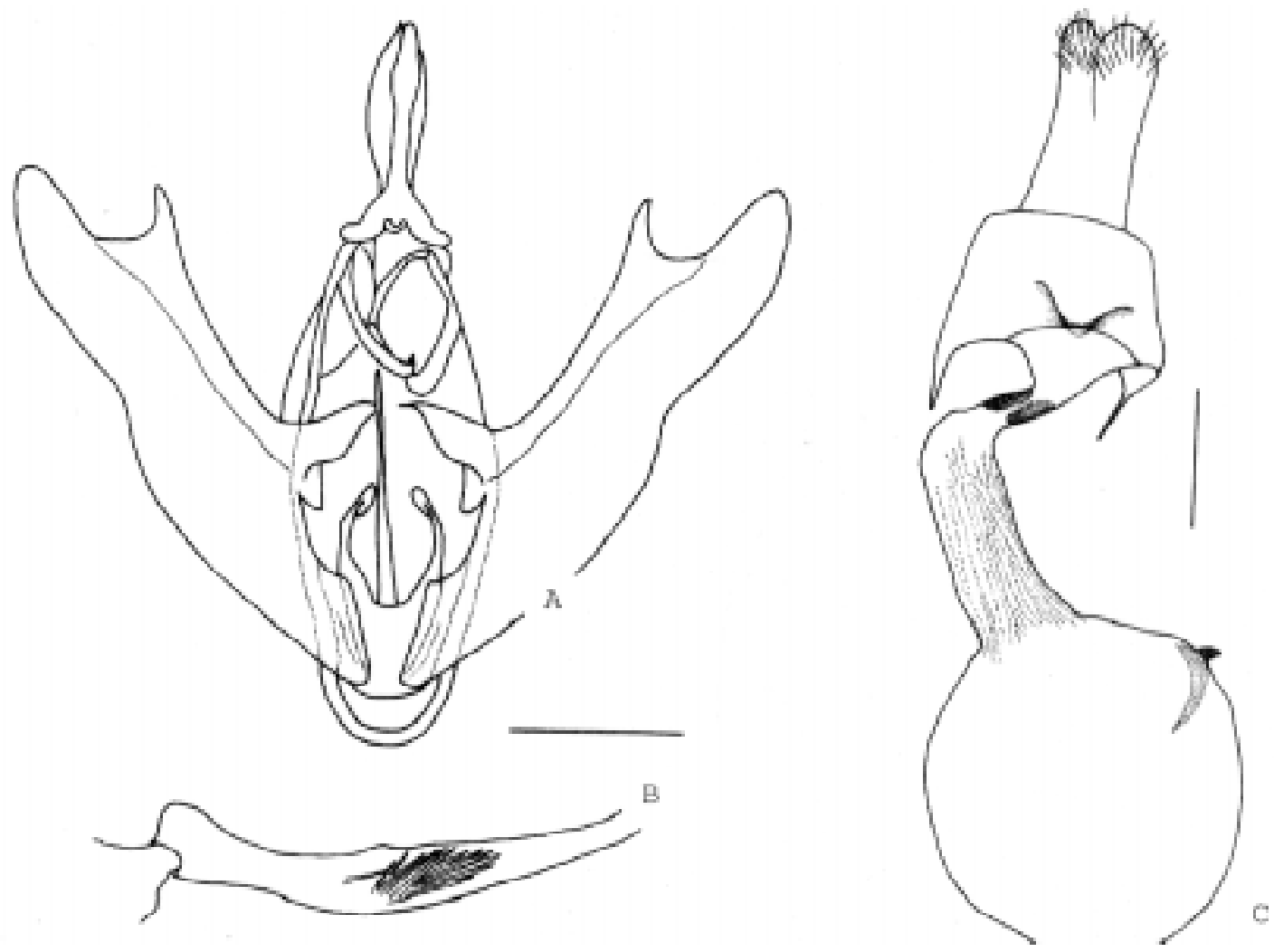

Fig. 5: Armaduras genitales del macho y la hembra de H. bartletti: (A) genitalia del macho en vista ventral; (B) aedeagus en vista lateral; (C) genitalia de la hembra en vista lateral. Escala: $1 \mathrm{~mm}$.

Male and female genitalia of $H$. bartletti: (A) male genitalia in ventral view; (B) aedeagus in lateral view; (C) female genitalia in lateral view. Scale: $1 \mathrm{~mm}$. 
Hasodima boreas (Butler 1882) comb. nov. (Fig.1C, $4 A$ y $4 B$ )

Bacillogaster boreas Butler 1882 p. 391; Butler 1894, p. 519; Angulo \& Casanueva 1981, p. 19.; Scoble 1999, p. 674 (Oratha).

Tipo. Bacillogaster boreas, Sintipo O', Valparaíso, Chile, BMNH (examinado).

Diagnosis. H. boreas se distingue de las otras especies del género por el color oscuro de las alas anteriores y por las antenas simples en el macho. La genitalia del macho de $H$. boreas se reconoce por la furca tubular, pseudouncus en forma de gancho y la hendidura poco notoria en el ápice de las valvas (región del cucullus). Estas características también nos permiten distinguirla de las especies del género Aconcagua Rindge 1983, con las cuales comparte muchos caracteres externos.

Redescripción: macho (Fig. 1C). Cabeza: antenas simples, superficie dorsal cubierta con escamas castaño claras; frente y vértex castaño claros y con escamas castaño oscuro entremezcladas. Tórax cubierto por penachos de escamas castaño claras y oscuras cuyo borde posterior es blanco, superficie ventral y lateral castaño ceniciento; patagias castaño claras con el borde dorsal castaño oscuro; tégula con el margen anterior castaño oscuro, cuerpo castaño claro a ceniciento. Patas castaño claras; fórmula tibial 0-2-4. Alas anteriores castaño oscuras; bandas medial y postmedial castaño oscuras y limitadas por una franja más clara; banda basal negra entre la región costal y la vena Sc; banda terminal y mancha discal levemente marcada; ventralmente más claras, con la mancha discal más aparente. Alas posteriores ocre con una franja más oscura en el borde exterior. Ventralmente ocre más homogéneo. Abdomen abigarrado con escamas castaño oscuras y claras en su superficie dorsal y lateral, castaño ceniciento en la superficie ventral; penachos de escamas en el dorso de los segmentos abdominales 1 al 8, castaño claros hacia la base y oscuros hacia el margen distal; penachos de los segmentos abdominales 1 y 2 pequeños, ocupan $1 / 3$ del largo de cada segmento; penachos de otros segmentos ocupan la mitad de la longitud. Genitalia del macho (Fig. 4A y 4B). Uncus, curvo, de longitud igual a la mitad de la yuxta; pseudouncus en forma de gancho, subigual en tamaño del uncus; socii reducido, redondeado y con cerdas; gnathos en forma de "V", terminado en punta, no dentado; yuxta con zona basal en forma de ancla, redondeada en el centro; proceso del anellus único y centrado, con ensanchamiento apical en forma de abanico; saccus redondeado; valva sencilla, de contorno poco redondeado, terminada en una pequeña cúspide en el extremo superior interno, mide dos veces la longitud de la yuxta; aedeagus curvado, aristado en el extremo, de longitud algo menor de la mitad de la del anellus; vesica con una placa esclerosada cubierta de microespinas.

Hembra. desconocida.

Longitud del ala anterior. De 15 a $17 \mathrm{~mm}$ en los machos.

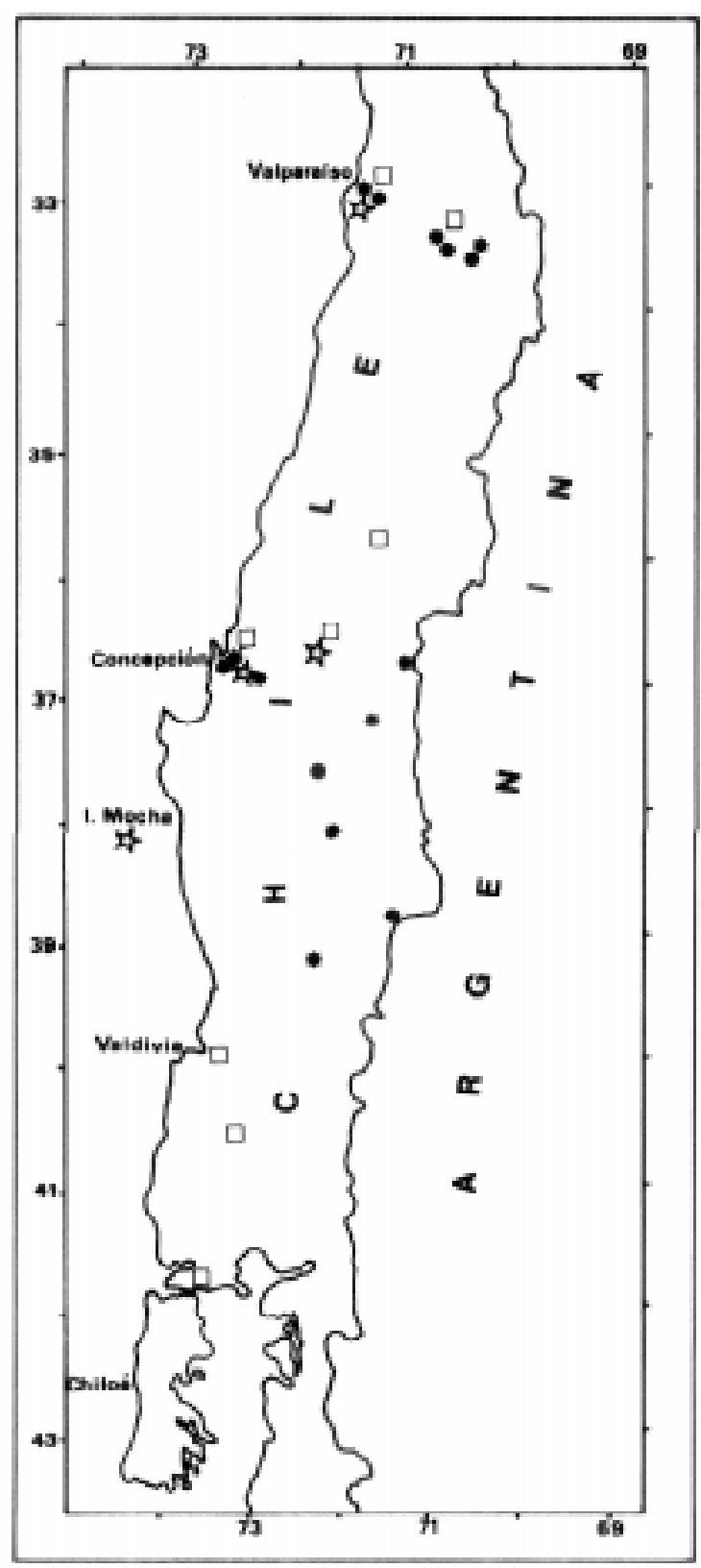

Fig. 6: Distribución de las especies de Oratha y Hasodima. Círculo blanco: O. significata; cuadrado blanco: $H$. elegans; círculo negro: H. boreas; y estrella: H. bartletti.

Distribution of the Oratha and Hasodima species. White circle: $O$. significata; white square: $H$. elegans; black circle: $H$. boreas; and star: H. bartletti. 
Distribución. Esta especie se distribuye entre $\operatorname{los} 33^{\circ}$ y $36^{\circ} \mathrm{S}$, entre la Provincia de Santiago y Concepción (Fig. 6).

Período de vuelo. Colectado desde el 9 de noviembre hasta el 25 de marzo.

Observaciones. La redescripción de la especie se basó en 20 machos, la hembra se desconoce. No se observó variación intraespecífica en los machos examinados. H. boreas entre las especies del género Hasodima es la más disímil en su apariencia externa. Externamente es muy parecida a las especies del género Aconcagua (Rindge 1983) de la tribu Nacophorini.

Material examinado (20 ejemplares). Valparaíso. Valparaíso, 1 Sintipo ${ }^{\prime}(\mathrm{BMNH})$. Santiago. Tobalaba: 10, 19-1-1947; 1 7 , 9-111947 (MZUC). Cordillera. Guayacán: $507,1-$ 1951; 2 ऊ', 12-1950 (MZUC); 1 đ', 18-12-1943 (MNHN). Concepción. Concepción: 1 12 , 12-31960; 1 $\sigma^{\prime}, 25-3-1961 ; 1 \sigma^{\prime}, 7-3-1960 ; 1 \sigma^{\circ}, 11-3-$ 1959; 1 $\sigma^{\gamma}, 14-3-1960 ; 10 \%$ 12-3-1961 (MZUC). Malleco. Icalma: 20' $\sigma^{\prime}, 6-2-1952$ (MZUC). Cautín. Choroico: 1 $\sigma^{7}$ 2-2-1989, Artigas coll. (MZUC).

Hasodima bartletti sp. nov. (Fig. 2D, 5A, 5B y $5 C)$

Tipo. Holotipo , Península de Hualpén, 9-32001, L.E. Parra coll. (MZUC); Alotipo $\bigcirc$, Península de Hualpén, 12-2-2001, L.E. Parra coll. (MZUC).

Diagnosis. Hasodima bartletti se distingue de las otras especies por su coloración mucho más clara (castaño ceniciento) de las alas anteriores y del cuerpo. En la genitalia del macho, el pseudouncus es lanceolado y la furca acicular. La porción anterior esférica del corpus bursae en la genitalia de la hembra distingue a esta especie de H. elegans.

Descripción: macho (Fig. 1D). Cabeza castaño cenicienta; antenas bipectinadas, castaño cenicientas. Tórax castaño ceniciento; patas similares en coloración, fórmula tibial 0-2-4. Alas anteriores de color castaño ceniciento; bandas medial, postmedial y terminal marcadas en castaño oscuro, al igual que la mancha discal; la banda postmedial se encuentra inclinada hacia la base; banda basal ancha y difuminada, castaño oscura; manchas castaño oscuro en la zona apical, esbozando la banda adterminal; vista ventral color castaño más homogéneo, mancha discal más tenuemente marcada; banda terminal castaño oscuro. Alas posteriores de color ocre, banda terminal castaño oscuro; mancha discal y banda medial en castaño claro; vista ventral ocre; banda medial y mancha discal más aparentes, banda terminal castaño oscuro. Abdomen de color similar a las alas, con penachos de escamas en el tercer segmento abdominal. Genitalia del macho (Fig. 5A y 5B). Uncus en forma de gancho; pseudouncus lanceolado; socii presente, con cerdas; gnathos en forma de "V" con un ensanchamiento final dentado y terminado en punta; yuxta subtriangular con dos procesos laterales de longitud igual a la proyección menor del uncus; furca acicular 2,5 veces más largas que los procesos laterales; valvas sencillas con una profunda escisión apical, una cúspide en el centro y provistas de numerosos pelos; saccus redondeado; aedeagus recto, ensanchado en la zona de la vesica; vesica armada con microespinas.

Hembra. Similar al macho, con antenas simples; tibias posteriores sin pincel de pelos. Genitalia de la hembra (Fig. 5C). Zona anterior del corpus bursae globosa y membranosa, zona posterior esclerosada con surcos longitudinales; signum grande, ensiforme cinco veces menor que el corpus bursae; apófisis anteriores cuatro veces menores que las apófisis posteriores; ductus seminalis nace en el límite entre el corpus bursae y el ductus bursae; ductus bursae con las áreas laterales esclerosadas, de forma cuadrangular, 10 veces menor que el corpus bursae.

Longitud del ala anterior. Medida desde la base al ápice: de 12 a $15 \mathrm{~mm}$ para los machos y de 12 a $16 \mathrm{~mm}$ para las hembras.

Distribución. Esta especie se distribuye en Chile Continental entre los $33^{\circ}$ y los $36^{\circ} 49^{\prime}$ S en la Isla Mocha, frente a las costas de la Provincia de Arauco (Fig. 6).

Periodo de vuelo. Especie recolectada desde el 26 de septiembre al 26 de marzo.

Etimología. La especie esta dedicada al naturalista William Bartlett-Calvert.

Observaciones. La descripción de esta especie se basó en 23 machos y 33 hembras. No se observó variación intraespecífica en los ejemplares estudiados.

Material examinado (56 ejemplares). Valparaíso. El Quisco: 1 PARATIPO, 12-111950 (MZUC). Ñuble. Chillán: 1 P Paratipo, 103-1960 (MZUC). Concepción. Península de Hualpén: 1 HOLOTIPO, 9-3-2001, L.E. Parra coll. (MZUC); $1 Q$ Alotipo, 12-2-2001, L.E. Parra coll. (MZUC); $2 O^{7}$ PARATIPOS, 9-3-2001, C. Pohl coll. (MZUC). Paratipos: San Pedro: 10, 10-10-1956, Cid col. Concepción: 1 J, 28-12$1951 ; 1 \bigcirc, 23-12-1958 ; 1 \bigcirc, 29-12-1958 ; 1 \bigcirc, 10-$ 1-1959; 2ᄋ०, 26-1-1959; 1 , 3-2-1959; 1 ○, 8-2$1959 ; 1 \bigcirc, 10-2-1959 ; 1 \bigcirc, 25-2-1959 ; 1 \bigcirc, 20-10-$ 1959; 1@, 2-2-1960; 1@, 3-3-1960; 1@, 4-3-1960; 
$1 \sigma^{7}, 7-3-1960 ; 1 \sigma^{7}, 30-1-1961 ; 3 \sigma^{7}, 11-2-1961$; 10 , 16-2-1961; 1ᄋ, 4-3-1961; 1Q, 6-3-1961; $10,7-11-1961 ; 10,18-12-1961 ; 10,28-12-1961$; $1 \bigcirc$ у $1 \bigcirc^{\gamma}, 11-1-1962 ; 1 \bigcirc^{\gamma}, 23-2-1961 ; 1 \bigcirc, 3-3-$ $1961 ; 1$, 3-1-1962; 1 \% 8-1-1962; 10, 25-1$1962 ; 10$, 12-2-1962; 10, 13-2-1962; 10, 26-31962; 10 , 2-2-1960. Dichato: 1Q, 14-1-1951. Arauco. Isla Mocha: 300, 26-9-1998, E. Mundaca coll.; 2QQ, 6-10-1998; 2Q९, 13-12-1998, E. Mundaca coll.; 300, 15-12-1998, E. Mundaca coll.; 2 $\sigma^{\top}, 18-12-1998$, E. Mundaca coll (MZUC).

\section{DISCUSIÓN}

Los géneros Oratha y Hasodima y sus respectivas especies poseen varios caracteres morfológicos consistentes, entre ellos, los caracteres derivados particulares que sugieren la monofilia para cada género, tres sinapomorfias en Oratha y una en Hasodima (ver monofilia más arriba).

Aunque las tribus Anagogini, Cingiliini y Ourapterygini tienen muchos caracteres en común en sus diferentes estados de desarrollo (McGuffin 1987), la presencia de una furca impar y simétrica en la yuxta de la genitalia del macho permite incluir los géneros Oratha y Hasodima en la tribu Ourapterygini.

Un análisis cladístico preliminar muestra que Hasodima y Oratha son géneros hermanos, y junto a Hemiloxia están representados en un mismo clado (L. Parra resultados no publicados). La sinapomorfía de Oratha y Hasodima es la presencia de un signum pedunculado. La hendidura en la región del cucullus en la valva de la armadura genital del macho es la sinapomorfía del clado: $(($ Oratha + Hasodima $)+$ Hemiloxia $))$. Las antenas pectinadas del macho y la presencia del signum en la genitalia de la hembra sugiere que los tres géneros anteriores están relacionados con Perusia Herrich-Schäffer.

La separación en distintos géneros y la proposición de nuevos taxa en Lepidoptera, frecuentemente se sugieren sobre la base de las diferencias morfológicas en la armadura genital del macho (Scoble 1986, Choi 1999). La distinción entre los géneros Oratha y Hasodima se basa fundamentalmente en este principio, ya que las estructuras morfológicas asociadas a la cabeza, cuerpo y alas de las distintas especies son prácticamente indistinguibles entre ambos géneros, mientras que la armadura genital entrega caracteres diagnósticos útiles en la diferenciación. En el caso de las armaduras genitales de las hembras, las diferencias son más sutiles, pero al mismo tiempo evidencian el parentesco que tienen las diferentes especies que pertenecen a un mismo género. En
Oratha, las estructuras asociadas al uncus, gnathos y furca de la yuxta, y en Hasodima, la presencia de un psudouncus, son los caracteres de la armadura genital del macho que permiten diferenciar ambos taxa.

Scoble (1999) incluye en Oratha a cuatro especies: Oratha significata, O. boreas, O. parva y $O$. sericea, después de este estudio constituye un género monotípico (O. significata), diferenciándose marcadamente, de otros taxa de la tribu Ourapterygini, por la morfología de la armadura genital del macho.

Según Scoble (1999), Hasodima (Butler 1882) alberga dos especies: H. elegans Butler, con distribución en Chile y H. melanoglyphica Dognin presente en Colombia. En este estudio sólo se ha considerado la especie presente en Chile (especie tipo del género), ya que no fue posible obtener el tipo ni ejemplares de la especie distribuida en Colombia. Sólo Oratha boreas comparte caracteres con la especie tipo del género Hasodima (presencia de pseudouncus, tipo de furca, gnathos en forma de "V"), por lo que esta especie ha sido incluida en este género.

Posición sistemática de Oratha parva (Butler) y O. sericea (Butler) sensu Scoble (1999)

Los ejemplares de Bacillogaster parva Butler 1882 presentan características de maculación y de la genitalia que corresponden a la especie Aconcagua fessa descrita por Rindge (1975), por lo que parva es excluida del género Oratha e incorporada al género Aconcagua Rindge (1983), de la tribu Nacophorini. B. parva Butler 1882 es el sinónimo senior de A. fessa Rindge 1975, por lo tanto se propone como especie válida a Aconcagua parva (Butler) comb. nov. y a A. fessa Rindge como un nuevo sinónimo de ésta. La posición sistemática de parva, propuesta aquí, se basa en la revisión del ejemplar tipo de parva y la información proporcionada por Rindge $(1975,1983)$.

Oratha sericea (sensu Scoble 1999), presenta caracteres que por el momento no permite su inclusión en ninguno de los géneros revisados. La armadura genital del macho tiene la transtilla muy desarrollada, en forma de gancho, en su borde interno con dientes dispersos. Probablemente este taxon sea un representante de la tribu Nacophorini.

\section{AGRADECIMIENTOS}

Agradecemos a Mario Elgueta y Ariel Camousseight (MNHN), Jorge N. Artigas (MZUC) 
y Enrique Mundaca por proveer parte del material utilizado en este estudio. Malcolm J. Scoble $(\mathrm{BMNH})$ por patrocinar la estadía de L.E. Parra en The Natural History Museum. M. PasculaToca fue becaria de Intercampus, AEGCI España en el período septiembre - octubre de 1999. Finalmente a los proyectos 98.113.047-1D1 y 200.113.056-1.0 de la Dirección de Investigación, Universidad de Concepción, por el financiamiento de esta investigación.

\section{LITERATURA CITADA}

ANGULO AO \& ME CASANUEVA (1981) Catálogo de los lepidópteros geométridos de Chile (Lepidoptera: Geometridae). Boletín Sociedad de Biología de Concepción (Chile) 51: 7-39.

ARMESTO J, P LEÓN-LOBOS \& MK ARROYO (1996) Los bosques templados del sur de Chile y Argentina: una isla biogeográfica. En: Armesto J, C Villagrán \& MK Arroyo (eds) Ecología de los bosques nativos de Chile. 23-28 pp. Editorial Universitaria, Santiago, Chile.

BUTLER AG (1882) Heterocerous Lepidoptera collected in Chili by Thomas Edmons, Esq. Part III Geometrides. Transactions of the Entomological Society of London 3: 339-423.

BUTLER AG (1894) Nuevos lepidópteros de Chile. Mariposas colectadas en Chile por el Señor Tomas Edmonds publicadas en Londres en los "Transactions of the Entomological Society" años 1881 a 1883. Segunda parte, Lepidoptera nocturna (continuación). Anales de la Universidad de Chile 86: 459-532. (traducción al español de W. Bartlett-Calvert)

CHOI SW (1999) Taxonomic review of a new genus, Diathera gen. n., from southeast Asia (Lepidoptera, Geometridae: Larentiinae). Journal of Natural History 33: 1039-1048.

FELDER C \& AF ROGENHOFER (1875) Reise der österreichischen Fregatte Novara um die Erde (Zoologischer Theil) Band 2 (Abtheilung 2): pls. 121-140. Wien.

FORBES WTM (1948) Lepidoptera of New York and neighboring states. Part II. Memoir Cornell University Agricultural Experimental Station 274:1-263.

FLETCHER DS (1979) Geometroidea. En: Nye IWB (ed) The generic names of moths of the world 3: 1-243. Trustees of the British Museum of Natural History, London, United Kingdom.
KLOTS AB (1970) Lepidoptera. En: Tuxen SL (ed) Taxonomist's glossary of genitalia in insects (second edition): 115-130. Munksgaard, Copenhagen, Denmark.

McGUFFIN WC (1987) Guide to the Geometridae of Canada (Lepidoptera). II. Subfamily Ennominae. 4. Memoirs of the Entomological Society of Canada 138: 1-182.

PARRA LE (1997) Revisión de los Lithinini y Trichopterygini (Lepidoptera: Geometridae) de Chile y de la región andina argentina adyacente. Tesis de Doctorado, Universidad de Oviedo, Oviedo, España. $469 \mathrm{pp}$.

PARRA LE (1999a) Revision of the Neotropical genus Psilaspilates (Lepidoptera: Geometridae). Annals of the Entomological Society of America. 92: 460-472.

PARRA LE (1999b) Revisión del género Euclidiodes Warren, 1895 (Lepidoptera: Geometridae). Revista Chilena de Historia Natural 72: 643-659.

RINDGE FH (1975) A revision of the moth genus Ceratonyx (Lepidoptera, Geometridae). American Museum Novitates 2564: 1-30.

RINDGE FH (1983) A generic revision of the new world Nacophorini (Lepidoptera, Geometridae). Bulletin of the American Museum of Natural History 175: 147 262.

RINDGE FH (1986) Generic descriptions of New World Lithinini (Lepidoptera, Geometridae). American Museum Novitates 2838: 1-68.

SCOBLE MJ (1986) The structure and affinities of the Hedyloidea: a new concept of the butterflies. Bulletin of the British Museum of Natural History 53: 251286.

SCOBLE MJ (1995) The Lepidoptera: form, function and diversity. The Natural History Museum \& Oxford University Press, London, United Kingdom. 404 pp.

SCOBLE MJ (1999) Geometrid moths of the world: a catalogue (Lepidoptera, Geometridae). British Museum of Natural History, London, United Kingdom. $1016 \mathrm{pp}$.

VILLAGRÁN C \& LF HINOJOSA (1997) Historia de los bosques de sur de Sudamérica, II: análisis fitogeográfico. Revista Chilena de Historia Natural 70: 241-267.

WALKER F (1863) List specimens of Lepidoptera. Insect Collection of the British Museum 27: 15.

Editor Asociado: Luis Ebensperger

Recibido inicialmente el 4 de septiembre de 2001; segundo proceso de evaluación iniciado el 27 de diciembre de 2002; aceptado el 27 de enero de 2003 


\section{ANEXO 1 \\ Clave para identificación de adultos del género Hasodima Butler \\ Identification key to adults of genus Hasodima Butler}

1. Alas anteriores castaño claras, verdosas o castaño cenicientas, bandas medial y postmedial castaño oscuras; antenas del macho pectinadas; pseudouncus lanceolado o romboídeo; furca espiniforme 2

1 'Alas anteriores castaño oscuras, bandas medial y postmedial notorias por su coloración clara hacia el margen anal del ala (Fig. 1C); antenas del macho simples; pseudouncus en forma de gancho; furca subcilíndrica (Fig. 4A) .

H. boreas (Butler) comb. nov.

2. Alas anteriores castaño claras a verdosas, área entre bandas medial y postmedial más oscura (Fig. 1b); pseudouncus romboídeo; furca espiniforme, corta, no alcanza la transtilla (Fig. 3a); porción anterior del corpus bursae subcónico (Fig. 3C) H. elegans Butler

$2^{`}$. Alas anteriores castaño cenicientas, mancha discal notoria, castaño oscura (Fig. 1D); pseudouncus lanceolado; furca acicular, delgada y larga, sobrepasa la transtilla (Fig. 5A); porción anterior del corpus bursae esférico (Fig. 5C) .... 